\title{
Element-selective ultrafast magnetization dynamics of hybrid Stoner-Heisenberg magnets
}

\author{
Mohamed F. Elhanoty $\odot,{ }^{1, *}$ Olle Eriksson, ${ }^{1,2}$ Ronny Knut, ${ }^{1}$ Olof Karis, ${ }^{1}$ and Oscar Grånäs ${ }^{1, \dagger}$ \\ ${ }^{1}$ Division of Materials Theory, Department of Physics and Astronomy, Uppsala University, Box-516, SE 75120 Uppsala, Sweden \\ ${ }^{2}$ School of Science and Technology, Örebro University, SE-701 82 Örebro, Sweden
}

(Received 30 November 2021; revised 7 February 2022; accepted 18 February 2022; published 2 March 2022)

\begin{abstract}
Stoner and Heisenberg excitations in magnetic materials are inherently different. The first involves an effective reduction of the exchange splitting, whereas the second comprises excitation of spin waves. In this work, we test the impact of these two excitations in the hybrid Stoner-Heisenberg system of FePd. We present a microscopic picture of ultrafast demagnetization dynamics in this alloy, which represents both components of strong local exchange splitting in $\mathrm{Fe}$ and induced polarization in Pd. We identify the spin-orbit coupling (SOC) and the optical intersite spin transfer (OISTR) as the two dominant factors for demagnetization at ultrashort timescales. Remarkably, the drastic difference in the origin of the magnetic moment of the Fe and Pd species is not deciding the initial magnetization dynamics in this alloy. By tuning the external laser pulse, the extrinsic OISTR can be manipulated for site-selective demagnetization on femtosecond timescales providing the fastest way for optical and selective control of the magnetization dynamics in alloys. Saliently, our results signify why various experiments demonstrating OISTR might obtain conflicting results.
\end{abstract}

DOI: 10.1103/PhysRevB.105.L100401

\section{INTRODUCTION}

Laser-induced ultrafast magnetization dynamics (LIUMD) has been demonstrated to be an efficient way to manipulate the spin and orbital moments of magnetic elements in the femtosecond regime [1,2]. Manipulation of the demagnetization amplitude and the corresponding timescale in an elementspecific way, for multicomponent systems, is of fundamental interest to further engineer the LIUMD technique [3-6]. Although the experimental realizations are well established the theory is lagging behind. Several theoretical attempts to explain the interplay between various degrees of freedom in the demagnetization process have been suggested [7-18], all, however, showing deficiencies in explaining the full experimental results.

Most systems that have been investigated so far, both by experiments and theory, are known to be well represented by a Heisenberg Hamiltonian, possibly with a correction from the anisotropic exchange (e.g., the Dzyaloshinskii-Moriya interaction) [19]. At longer timescales, e.g., those that are relevant for magnon excitations, these systems can be interpreted from a low-energy spin-Hamiltonian, and this has motivated an analysis of the ultrafast experiments reported in Ref. [2] by means of atomistic spin-dynamics $[16,19,20]$. However,

\footnotetext{
*mohamed.elhanoty@physics.uu.se

†Corresponding author: oscar.granas@physics.uu.se
}

Published by the American Physical Society under the terms of the Creative Commons Attribution 4.0 International license. Further distribution of this work must maintain attribution to the author(s) and the published article's title, journal citation, and DOI. Funded by Bibsam. magnetic systems that are known to have Stoner excitations are much less studied. A key to understanding the LIUMD process, in general, is to compare the excitation processes in Heisenberg and Stoner systems on equal footing.

Time-dependent density functional theory (TDDFT) is an $a b$ initio method that can be tailored to study LIUMD, where only the composition and geometry of the material are needed as input, in combination with the experimental parameters for the laser pulse [21]. Both the relevant macroscopic experimental observables, such as magnetization, and microscopic details, such as orbital and spin-resolved particle occupations, are extracted from TDDFT calculations, for instance, as implemented in the ELK code [22]. The adiabatic local spin density approximation (ALSDA) of the exchange and correlation functional showed significant success in addressing the fundamental questions of the early parts of the magnetization dynamics with very good agreement with the transient magneto-optic Kerr effect (MOKE) and x-ray magnetic circular dichroism (XMCD) experiments [3,14,14,23-27]. In these studies, spin-flips mediated by SOC, and their importance for the OISTR effect, were suggested to be the main source of demagnetization, at least for the early parts of the magnetization dynamics.

Elemental Pd is on the verge of being ferromagnetic due to its high density of states (DOS) at the Fermi level [28] and a Stoner product just below 1. Alloys between Pd and magnetic $3 d$ elements such as Fe or Co represent unconventional magnets, with an experimentally observed and theoretically calculated "giant" magnetic moment [29-31]. This giant moment, in the dilute limit, stems from an enhanced local moment of the $\mathrm{Co}$ and $\mathrm{Fe}$ atoms, compared to their elemental values [30], in combination with a cloud of induced moments of the Pd atoms that are in the vicinity of the impurity host. 
One can therefore conclude that alloys between magnetic $3 d$ elements $(\mathrm{Cr}, \mathrm{Mn}, \mathrm{Fe}, \mathrm{Co}$, and $\mathrm{Ni}$ ) and $\mathrm{Pd}$, especially in the dilute limit, are ideal to investigate the phenomenon of LIUMD of a multicomponent system with building blocks that have drastically different magnetic properties and mechanisms of excitations. In fact, one experimental investigation of light-induced modification of the magnetism was published for FePd [32], but element-specific analysis of the magnetism was not made in this work, nor was the expected difference in excitation properties analyzed.

The discussion above motivates an investigation of the element-specific magnetization of FePd alloy using TDDFT. We chose $\mathrm{FePd}_{3}$ as a representative because it contains the essential features of combining Stoner and Heisenberg excitations in the same compound while providing a computationally manageable system. In making this choice, one also mixes elements with expected different site-projected electronic structures, as well as different SOC strengths. As we shall see below, the intrinsic and extrinsic timescales of the SOC and OISTR, respectively, allow studying the dependency of the OISTR process on the pump laser parameters giving rise to possible inconsistency in experiments comprising OISTR. A general approach to tailor the laser parameters for an element-selective and efficient control of the magnetization dynamics in alloys at an unprecedentedly short timescale is also demonstrated.

\section{METHODOLOGY}

TDDFT is an extension of the ground-state (GS) DFT formalism, through the one-to-one mapping between the external potential $v_{\text {ext }}(\mathbf{r}, t)$ and the electronic density $n(\mathbf{r}, \mathrm{t})$ that only depends on spatial coordinates in the GS, while on both spatial coordinates and time in TDDFT. This unique one-toone correspondence allows for a fully interacting system to be mapped into an equivalent non-interacting one with a time-dependent Kohn-Sham (KS) effective potential $v_{s}(\mathbf{r}, \mathrm{t})$, that produces the same density as the fully interacting system during every time propagation step. Notably, $v_{s}(\mathbf{r}, t)$ is a sum of three terms $v_{s}(\mathbf{r}, t)=v_{\text {ext }}(\mathbf{r}, t)+v_{H}(\mathbf{r}, t)+v_{\mathrm{xc}}(\mathbf{r}, t)$, where $v_{\text {ext }}(\mathbf{r}, t)$ is the external potential, $v_{H}(\mathbf{r}, t)$ is the Hartree potential, and $v_{\mathrm{xc}}(\mathbf{r}, t)$ is the exchange-correlation $(\mathrm{XC})$ potential. Within TDDFT, the effect of an external laser pulse is treated within the dipole approximation. This approximation is valid for radiation with long wavelength compared to the lattice constant. For all pump-probe experiments done currently, this is a very good approximation $[10,14,17,18,25,26]$. This allows to consider only a time-dependent vector potential $\mathbf{A}_{\mathrm{ext}}(t)$ that modifies the kinetic energy (first term) of the Hamiltonian in Eq. (1)

$$
\begin{aligned}
& \left\{\frac{1}{2}\left(-i \nabla+\frac{1}{c} \mathbf{A}_{\mathrm{ext}}(t)\right)^{2}+v_{s}(\mathbf{r}, t)+\frac{1}{2 c} \sigma \cdot \mathbf{B}_{s}(\mathbf{r}, t)\right. \\
& \left.+\frac{1}{4 c^{2}} \sigma \cdot\left[\nabla v_{s}(\mathbf{r}, t) \times-i \nabla\right]\right\} \psi_{i}(\mathbf{r}, t)=\frac{\partial \psi_{i}(\mathbf{r}, t)}{\partial t},
\end{aligned}
$$

where $c$ is the speed of light, $\sigma$ is the Pauli matrix, and $\mathbf{B}_{s}(\mathbf{r}, t)$ is the effective KS magnetic field $\mathbf{B}_{s}(\mathbf{r}, t)=\mathbf{B}_{\text {ext }}(t)+$ $\mathbf{B}_{x c}(\mathbf{r}, t)$, where $\mathbf{B}_{\text {ext }}(t)$ is the magnetic field of the external laser pulse and $\mathbf{B}_{\mathrm{xc}}(\mathbf{r}, t)$ is the $\mathrm{XC}$-induced exchange splitting, expressed in the form of a field. The last term of Eq. (1) is the SOC term and $\psi_{i}(\mathbf{r}, t)$ is the two-component Pauli spinor. The atomic units are adopted in all the equations through this paper with $\hbar=e=m=1$.

The Hamiltonian of Eq. (1) is diagonalized in a linearized augmented plane wave (LAPW) basis set with two variational steps. In the first step, the Hamiltonian containing only the scalar potential is diagonalized in the LAPW basis. In the second variational step, a sufficient number of the resulting scalar states are used as a basis to construct the Hamiltonian with spinor degrees of freedom. The core electrons are treated within the radial Dirac equation and the valence electrons are treated within the relativistic Hamiltonian in the presence of the SOC.

The occupation of the KS transient state $\eta(\epsilon, t)$ discussed below is calculated by first projecting the TDKS state $\psi(r, t)$ on the time-independent, ground state $\phi(r)$ via

$$
P_{i j}^{\mathbf{k}}(t)=\int d^{3} r \phi_{i \mathbf{k}}^{*}(r) \psi_{j \mathbf{k}}(r, t) .
$$

Summing the square of the the projection $P_{i j}^{\mathbf{k}}(t)$ over all timedependent KS states, weighted by the occupation number $n_{j k}$, gives the TD occupation projected on the GS

$$
w_{i \mathbf{k}}(t)=\sum_{j} n_{j k}\left|P_{i j}^{\mathbf{k}}(t)\right|^{2} .
$$

Finally, the TD-projected DOS, $\eta(\epsilon, t)$, is evaluated according to

$$
\eta(\epsilon, t)=\sum_{i}^{\infty} \int_{\mathrm{BZ}} \delta\left(\epsilon-\epsilon_{i \mathbf{k}}\right) w_{i \mathbf{k}}(t),
$$

where $\epsilon_{i \mathbf{k}}$ is the $i$ th Kohn-Sham energy eigenvalue.

The structure is cubic with a $P m \overline{3} m$ space group. The calculations of this system were performed in a fully $a b$ initio and noncolinear fashion, as implemented in the ELK code, with $8 \times 8 \times 8 \mathbf{k}$-points in the Brillouin zone. The number of empty states per atom per spin is 20 and 30 for the data presented in Figs. 1 and 5, respectively. The calculations were performed in three steps. First, the geometry of the $\mathrm{FePd}_{3}$ system was optimized. Second, the GS of the optimized structure was determined and converged with respect to the number of empty states and k-points (see the Supplementary Materials [33]). Third, the tightly converged GS was evolved in time using Eq. (1) and a time-propagation scheme described in Ref. [34] with a time step of 2.4 attoseconds for the the data presented in Fig. 1 and 1.2 attoseconds for the data presented in Fig. 5 (see the Supplementary Materials [33]). A laser pulse with wavelength of $800 \mathrm{~nm}$, fluence of $1 \mathrm{~mJ} / \mathrm{cm}^{2}$, and full width at half maximum (FWHM) of $40 \mathrm{fs}$ was allowed to interact with the electronic subsystem and the response to this external field was followed for over $140 \mathrm{fs}$.

The local spin density approximation (LSDA) and its time-dependent adiabatic version (ALSDA) for exchange and correlation were used in the GS and time evolution calculations, respectively. Nevertheless, other approximations and developments to improve the treatment of the noncolinearity of the spin moments, correlation, and the relativistic effects in the exchange and correlation functional of Eq. (1) is desirable [35-37]. Secondary scattering processes such as the nuclei 


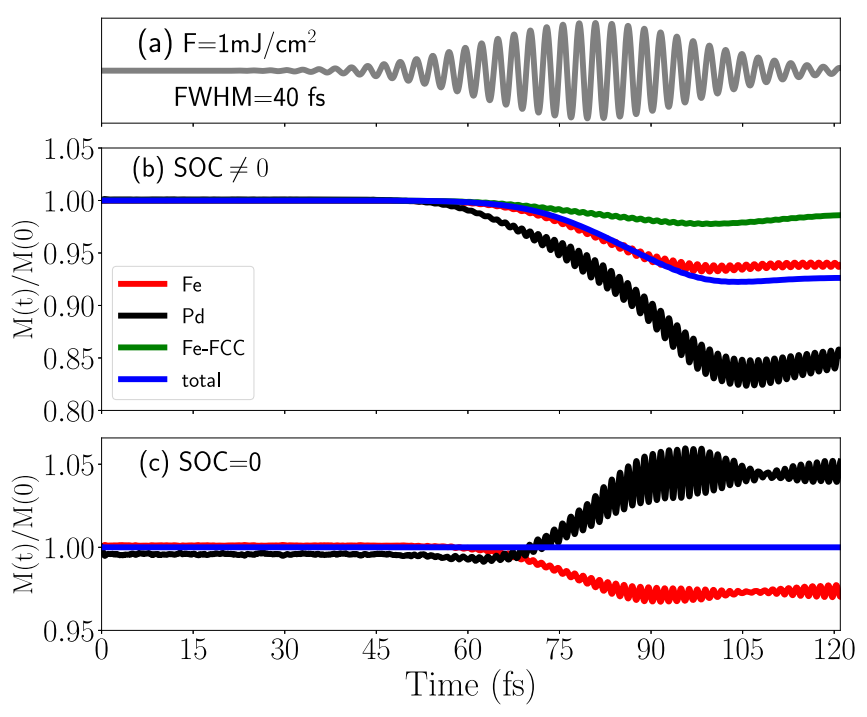

FIG. 1. (a) Pulse shape of the laser with FWHM $=40$ fs. Comparison between the $\mathrm{Fe}$ and $\mathrm{Pd}$ moments in $\mathrm{FePd}_{3}$ alloy and for elemental $\mathrm{Fe}$ with $\mathrm{FCC}$ structure with $\mathrm{SOC} \neq 0$ in (b) and $\mathrm{SOC}=0$ in (c) (see the text).

and radioactive contribution to dynamics were ignored. These effects are assumed to not influence the demagnetization at the early times of the process, as the dynamics is mainly driven by the electronic degrees of freedom coupled to the laser pulse [38-40].

\section{RESULTS}

The calculated total GS magnetic moment of the system is $4.18 \mu_{B}$, distributed as $3.23 \mu_{B}$ on $\mathrm{Fe}$ and $0.32 \mu_{B}$ on every $\mathrm{Pd}$ atom. The driven magnetization dynamics is shown in Fig. 1(b), where we compare the relative demagnetization of $\mathrm{Fe}$ (red graph) and $\mathrm{Pd}$ (black graph) of $\mathrm{FePd}_{3}$ to that of elemental Fe in the FCC structure (red graph) using the same laser pulse parameters [the pulse is illustrated in Fig. 1(a)]. The choice of FCC Fe as a comparison material, instead of $\mathrm{BCC} \mathrm{Fe}$, is motivated from geometrical reasons since replacing all $\mathrm{Pd}$ atoms with $\mathrm{Fe}$ in $\mathrm{FePd}_{3}$ results in the $\mathrm{FCC}$ structure (note that for FCC Fe, we obtain GS magnetic moments of $2.77 \mu_{B}$ per atom). The total driven demagnetization in Fig. 1(b) (blue line) is in a very good agreement with the one from the pump probe experiment reported in Ref. [32], where $\approx 7 \%$ of the total moment is lost at the first $\approx 100 \mathrm{fs}$.

Figure 1(b) shows that $\mathrm{Fe}$ and $\mathrm{Pd}$ atoms in $\mathrm{FePd}_{3}$ demagnetize very differently. The relative demagnetization of $\mathrm{Pd}$ (black graph) is much greater than that of Fe (red graph). Figure 1(b) also shows that the relative change of the Fe moment (red graph) in $\mathrm{FePd}_{3}$ is significantly larger, compared with the loss of the Fe moment in elemental Fe (green graph). To further shine the light on the mechanisms that are responsible for the LIUMD effect of $\mathrm{FePd}_{3}$, we performed calculations in which SOC was neglected. Interestingly, as Fig. 1(c) shows, the light-induced moment of Pd (black graph) is then found to increase, while the Fe moment decreases (red graph), seemingly in a similar way as that of elemental Fe (note the total (a)

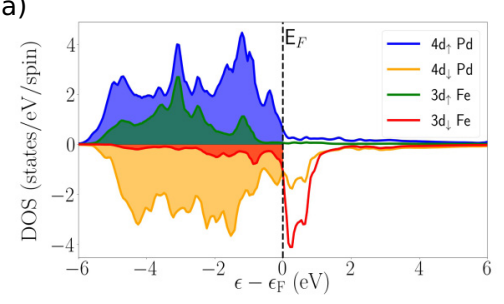

(b)

FIG. 2. (a) GS DOS (full lines) and occupations (shaded colors) of the $4 d$ and $3 d$ levels for the three $\mathrm{Pd}$ atoms and $\mathrm{Fe}$, respectively. (b) Schematic representations for the available source of demagnetization in FePd alloy.

moment (blue line) in Fig. 1(c) does not change since the SOC is turned off).

Remarkably, the amount of Fe demagnetization in calculations where SOC is included in Fig. 1(b) is significantly greater than for calculations when $\mathrm{SOC}=0$ in Fig. 1(c). Notably, the role of SOC on the demagnetization of $\mathrm{Pd}$ is more profound than for Fe. Pd is seen to lose about $15 \%$ of its initial moment when SOC is included while its moment increases if SOC is neglected. The magnetic moments in $\mathrm{FePd}_{3}$ hence demagnetize through two channels: the OISTR mechanism, which is most clearly seen from calculations without SOC, and from spin-flip transitions, visible from calculations with finite SOC. These two contributions are schematically shown in Fig. 2(b).

To analyze the results in Figs. 1(b) and 1(c), we inspect the PDOS curves, shown in Fig. 2(a) [41-43]. It should be noted that the unoccupied states of the minority spin channel (spin down, $\downarrow$ ) have special significance since they have been discussed to be relevant for the OISTR mechanism [26]. Figure 2(a) shows both the occupied and the unoccupied states for the $4 d$ and $3 d$ levels of the $\mathrm{Pd}$ and Fe atoms. The $3 d_{\downarrow}$ states of $\mathrm{Fe}$ (red graph) can be seen to have a significant amount of unoccupied states. These are states available for the OISTR mechanism that can be thought of as optical excitations (in the dipole approximation). Significantly fewer states are available for the unoccupied $4 d_{\downarrow}$ states of Pd (yellow graph). A pure OISTR process is best illustrated by the data in Fig. 1(c), where SOC coupling is neglected since spin-flip transitions are absent. Optical excitations then happen within each spin channel separately. As Fig. 2(a) shows, optical excitations are more efficient in the spin-down channel since the number of unoccupied states available in this process is larger. Figure 2(a) also shows that the occupied spin-down states have more pure Fe character while the unoccupied states have mixed, hybridized Fe and Pd character. The optical excitations can therefore be seen as transitions from the occupied minority spins of Pd to the unoccupied minority spin channel of Fe. This leads to a decrease of the Fe moment and an increase of the Pd moment, as shown in Fig. 1(c). Note that this process, as discussed also in Ref. [15], has to occur for hybridized states since otherwise dipole transitions would not be allowed.

To analyze further the microscopic origin of the OISTR process in element specific fashion, the time-dependent change in the majority (spin up $\uparrow$ ) and minority (spin down $\downarrow)$ spin occupations of the $d$ level, $\eta_{d}(t)$, is calculated using Eq. (4). The difference between $\eta_{d}(t)$ at $t=90 \mathrm{fs}$ and $t=0$ 
(a)

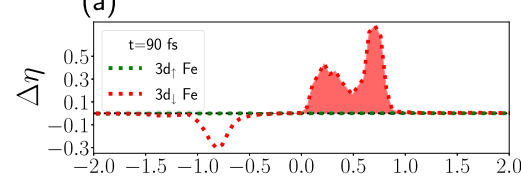

(c)

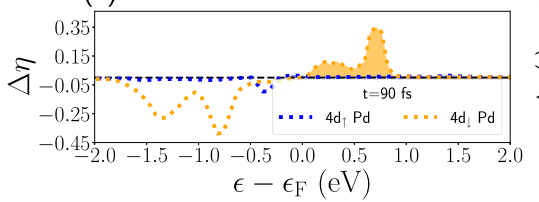

(b)

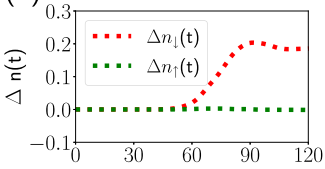

(d)

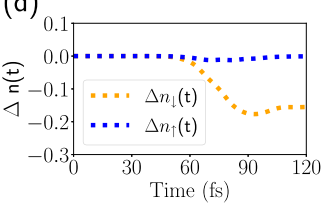

FIG. 3. Calculated results with SOC ignored. The change in the occupation of the $3 d$ up and down spin channels to the GS $[\eta(0)]$ at $t=90 \mathrm{fs}$ for $\mathrm{Fe}$ and $\mathrm{Pd}$ in (a,c), respectively. The change in the integration of the majority $\left(\Delta n_{\uparrow}(\mathrm{t})=\int d \epsilon\left[\eta_{\uparrow}(\epsilon, t)-\eta_{\uparrow}(\epsilon, 0)\right]\right)$ and minority $\left[\Delta n_{\downarrow}(t)\right]$ spins occupations over the entire energy range in time for Fe and Pd in (b,d), respectively.

$\left[\eta_{d}(0)\right]$ is depicted in Figs. 3(a) and 3(c) and 4(a) and 4(c) for $\mathrm{SOC}=0$ and SOC $\neq 0$, respectively. The integration of this quantity over the entire energy range $\left(\Delta n(t)=\int d \epsilon[\eta(\epsilon, t)-\right.$ $\left.\left.\left.\eta_{(} \epsilon, 0\right)\right]\right)$ is shown as function of time in Figs. 3(b) and 3(d) for $\mathrm{SOC}=0$ and in Figs. 4(b) and 4(d) for SOC $\neq 0$.

For the SOC $=0$ case, the decrease in the initially occupied minority spins of Pd [unshaded yellow area of Fig. 3(c)] and Fe [unshaded red area of Fig. 3(a)] is transferred to the initially unoccupied minority spin channel of Fe [shaded red area of Fig. 3(a)] and Pd [shaded yellow area of Fig. 3(a)], respectively. This can be pinpointed as a pure OISTR effect because SOC is turned off. The amount of the transferred spins in this situation is consistent with the GS empty states available in the minority spin channels for every species of Fig. 2(a). Note that $\Delta n_{\downarrow}(t)$ in Figs. 3(b) and 3(d) are also consistent with this observation. That is to say the OISTR process of transferring electron states from $\mathrm{Pd}$ to $\mathrm{Fe}$ and vice versa is selective only through the minority spin channel. For SOC $\neq 0$ case in Fig. 4 , in addition to OISTR, a signature of spin-flip transitions in the spin-up channel for both $\mathrm{Fe}$
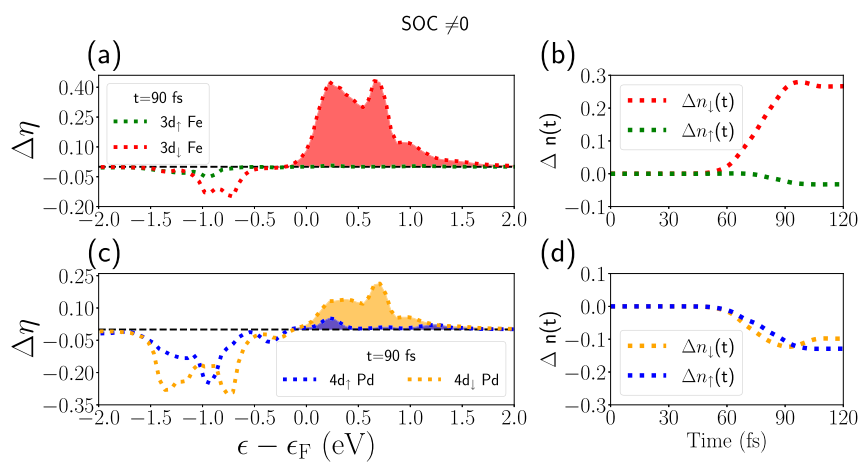

(d)

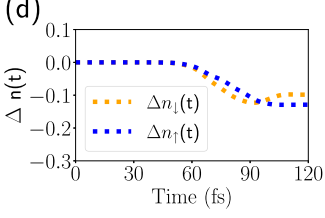

FIG. 4. Calculations with finite SOC. The change in the occupation of the $3 d$ up and down spin channels to the GS $[\eta(0)]$ at $t=90 \mathrm{fs}$ for $\mathrm{Fe}$ and $\mathrm{Pd}$ in $(\mathrm{a}, \mathrm{c})$, respectively. The change in the integration of the majority $\left(\Delta n_{\uparrow}(t)=\int d \epsilon\left[\eta_{\uparrow}(\epsilon, t)-\eta_{\uparrow}(\epsilon, 0)\right]\right)$ and minority $\left[\Delta n_{\downarrow}(t)\right]$ spins occupations over the entire energy range in time for Fe and $\mathrm{Pd}$ in $(\mathrm{b}, \mathrm{d})$, respectively.
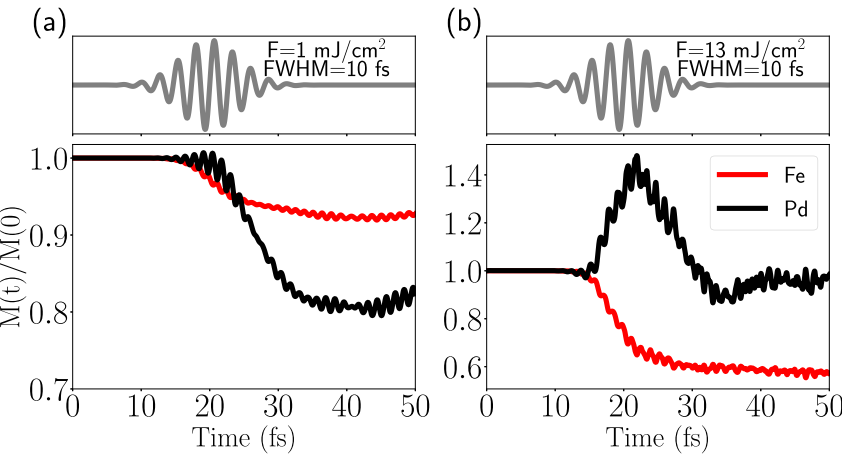

FIG. 5. (a) Pulse shape of the $1 \mathrm{~mJ} / \mathrm{cm}^{2}$ laser with $\mathrm{FWHM}=$ $10 \mathrm{fs}$ (upper panel). (b) Pulse shape of the $13 \mathrm{~mJ} / \mathrm{cm}^{2}$ with FWHM = $10 \mathrm{fs}$ (upper panel). The lower panels show the moment loss in Fe and Pd under the influence of the corresponding pulse.

[unshaded green line in Fig. 4(a)] and Pd [unshaded blue line in Fig. 4(c)] is manifested. The spin flips by SOC are more intense in $\mathrm{Pd}$ due to its stronger SOC.

\section{STRONG AND SHORT PULSES}

A striking feature in Fig. 1(b) with finite SOC is that the onset of the Pd demagnetization (black graph) comes earlier than the onset of Fe demagnetization (red graph). While the timescale at which SOC starts to cause demagnetization is an intrinsic property, the timescale at which OISTR can be activated is an extrinsic property, i.e., it can be controlled by the laser pulse parameters. Having identified OISTR and SOC as the two major sources of the demagnetization in FePd alloy, a relevant question is whether one can utilize the laser pulse parameters to selectively manipulate the onset and efficiency of demagnetization in an element-specific way. This question is crucial to unambiguously identify the fingerprints of OISTR in magnetic alloys. To investigate this premise, we considered a laser pulse with the same fluence as that considered in Fig. 1, but with a shorter FWHM. We also considered significantly larger fluences up to $13 \mathrm{~mJ} / \mathrm{cm}^{2}$ to amplify the OISTR effect.

Figure 5 shows results from calculations in which SOC was finite with FWHM $=10 \mathrm{fs}$ and fluence of $1 \mathrm{~mJ} / \mathrm{cm}^{2}$ [Fig. 5(a)] and stronger pulse of $13 \mathrm{~mJ} / \mathrm{cm}^{2}$ [Fig. 5(b)]. In both cases of Fig. 5, Fe is seen to have an onset of demagnetization that comes earlier than $\mathrm{Pd}$. For the strongest pulse [Fig. 5(b)], the moment on Pd actually increases initially, by $40 \%$, before it decreases and becomes reduced with respect to its GS value. This observation is in contrast to the results displayed in Figs. 5(a) and 1(b) (black graph). Although the signature of OISTR is diminished with the dominating SOC in Fig. 5(a) due to the low fluence, it is clearly manifested with higher fluence and shorter laser pulse as displayed in Fig. 5(b). Thus, for experiments demonstrating OISTR in magnetic alloys, the duration and the fluence of the pump laser together with the experimental measurement time become crucial parameters. This entails that experimental measurements confirming an observation of the OISTR effect, like in Refs. [3,23,24], should exhibit a similar trend of Figs. 5(b) and 5(a) by lowering the pulse power in which SOC competes and even diminishes the OISTR fingerprint. This also 
implies that possible discrepancies in demonstrating OISTR experimentally, e.g., increase or decrease of Pd moment, may emerge if the roles of the laser fluence and pulse duration are ignored.

The demagnetization of Fe in Fig. 5(b) is more efficient than its demagnetization in Fig. 1(b) and the previously studied case in Ref. [44]. While we obtain $\approx 40 \%$ of Fe moment reduction in Fig. 5(b), only $\approx 12 \%$ was obtained in Ref. [44] with even stronger and shorter pulses. OISTR is therefore regarded as an auxiliary approach to efficiently demagnetize the strong local moment of the stoner magnets, e.g., Fe, $\mathrm{Co}$, and $\mathrm{Ni}$ by alloying them with atoms that have nearly filled spin-down states of the $d$ orbital and utilization of ultrashort and strong laser pulses. Efficient demagnetization at the ultrashort timescale is desirable for the spintronics technology [4-6].

The trend exhibited by the three sets of calculations is rather clear. For the strongest pulse [Fig. 5(b)], one can understand this trend by noting $\mathbf{A}_{\mathrm{ext}}(t)$ of the laser pulse in Eq. (1) operates during short time and spin-flip transitions mediated by the intrinsic timescale of SOC become unimportant compared to the dominating OISTR effect. For this reason, the results in Fig. 5(b) are similar to the data without SOC in Fig. 1(c). Gradually decreasing the strength of $\mathbf{A}_{\text {ext }}(t)$ diminishes the importance of the OISTR effect, compared to spin-flip transitions. That is why in Fig. 5(a) the Pd moment stays constant initially, while the Fe moment decreases. For weaker and longer pulses in Fig. 1(b), in which the vector potential $\mathbf{A}_{\mathrm{ext}}(t)$ is less influential, Pd demagnetizes before Fe with a relative reduction of the moment that is strongest for Pd.

\section{CONCLUSION}

Remarkably, the drastic difference in the origin of the magnetic moment of $\mathrm{Fe}$ and $\mathrm{Pd}$ species is not a determining factor for the magnetization dynamics at the first $100 \mathrm{fs}$. Interestingly, the LIUMD of this hybrid Stoner-Heisenberg system, at the first $100 \mathrm{fs}$, is unexpectedly dominated by the OISTR effect in conjunction with spin flips mediated by the SOC.
The demagnetization of Pd was found to be mainly driven by its strong SOC, while in Fe both SOC and OISTR provide significant channels of demagnetization. The rigidity of the Fe moment, resulting from the strong intraatomic exchange coupling on $\mathrm{Fe}$, inhibits local spin-flip excitations $\left(J_{\mathrm{ex}} \gg\right.$ SOC), and the efficiency of the demagnetization process is strongly influenced by the ability to transfer spin density from the nearby Pd atom. Magnetization dynamics on longer timescales are bound to be influenced by magnon excitations and we suggest that at such timescales the known difference in processes determining magnetic excitations should play a role in the dynamics $[16,17,19,20,45]$. We also suggest that the nonequilibrium magnetization density thermalizes through magnon excitations, where the local exchange splitting is allowed to relax.

The intrinsic and extrinsic nature of the timescales of SOC and OISTR, respectively, allows to resolve potential contradictions in the measurements of magnetic alloys comprising OISTR by tuning the pump laser. Saliently, our findings entail that experiments measuring the OISTR effect should follow similar trends of Figs. 5(b) and 5(a) by reducing the pulse fluence in which SOC vies and even diminishes the OISTR signature. Also, our results highlight a general route for element selective and efficient manipulation of the demagnetization through an optical control of the laser pulse providing a fast and very efficient way to engineer the magnetization dynamics in alloys that is desirable for the spintronics technology.

\section{ACKNOWLEDGMENTS}

The computations were enabled by resources provided by the Swedish National Infrastructure for Computing (SNIC) at NSC and Uppmax partially funded by the Swedish Research Council through Grant Agreement No. 2018-05973. O.G. acknowledges financial support from the Strategic Research Council (SSF) Grant No. ICA16-0037 and the Swedish Research Council (VR) Grant No. 2019-03901. This work was also supported by the European Research Council Synergy Grant No. 854843 - FASTCORR.
[1] A. V. Kimel, A. Kirilyuk, and T. Rasing, Laser Photon. Rev. 1, 275 (2007).

[2] E. Beaurepaire, J.-C. Merle, A. Daunois, and J.-Y. Bigot, Phys. Rev. Lett. 76, 4250 (1996).

[3] M. Hofherr, S. Häuser, J. K. Dewhurst, P. Tengdin, S. Sakshath, H. T. Nembach, S. T. Weber, J. M. Shaw, T. J. Silva, H. C. Kapteyn, M. Cinchetti, B. Rethfeld, M. M. Murnane, D. Steil, B. Stadtmüller, S. Sharma, M. Aeschlimann, and S. Mathias, Sci. Adv. 6, eaay8717 (2020).

[4] C. D. Stanciu, F. Hansteen, A. V. Kimel, A. Kirilyuk, A. Tsukamoto, A. Itoh, and T. Rasing, Phys. Rev. Lett. 99, 047601 (2007).

[5] S. Wolf, D. Awschalom, R. Buhrman, J. Daughton, V. S. von Molnár, M. Roukes, A. Y. Chtchelkanova, and D. Treger, Science 294, 1488 (2001).

[6] I. Tudosa, C. Stamm, A. Kashuba, F. King, H. Siegmann, J. Stöhr, G. Ju, B. Lu, and D. Weller, Nature (London) 428, 831 (2004).
[7] B. Koopmans, H. Kicken, M. van Kampen, and W. de Jonge, J. Magn. Magn. Mater. 286, 271 (2005).

[8] B. Koopmans, J. J. M. Ruigrok, F. DallaLonga, and W. J. M. deJonge, Phys. Rev. Lett. 95, 267207 (2005).

[9] J. K. Dewhurst, S. Shallcross, P. Elliott, S. Eisebitt, C. v. K. Schmising, and S. Sharma, Phys. Rev. B 104, 054438 (2021).

[10] P. Elliott, N. Singh, K. Krieger, E. Gross, S. Sharma, and J. Dewhurst, J. Magn. Magn. Mater. 502, 166473 (2020).

[11] J. Hohlfeld, E. Matthias, R. Knorren, and K. H. Bennemann, Phys. Rev. Lett. 78, 4861 (1997).

[12] M. Aeschlimann, M. Bauer, S. Pawlik, W. Weber, R. Burgermeister, D. Oberli, and H. C. Siegmann, Phys. Rev. Lett. 79, 5158 (1997).

[13] E. Carpene, E. Mancini, C. Dallera, M. Brenna, E. Puppin, and S. De Silvestri, Phys. Rev. B 78, 174422 (2008).

[14] J. K. Dewhurst, P. Elliott, S. Shallcross, E. K. U. Gross, and S. Sharma, Nano Lett. 18, 1842 (2018). 
[15] P. Tengdin, C. Gentry, A. Blonsky, D. Zusin, M. Gerrity, L. Hellbrück, M. Hofherr, J. Shaw, Y. Kvashnin, E. K. DelczegCzirjak, M. Arora, H. Nembach, T. J. Silva, S. Mathias, M. Aeschlimann, H. C. Kapteyn, D. Thonig, K. Koumpouras, O. Eriksson, and M. M. Murnane, Sci. Adv. 6, eaaz1100 (2020).

[16] R. Chimata, L. Isaeva, K. Kádas, A. Bergman, B. Sanyal, J. H. Mentink, M. I. Katsnelson, T. Rasing, A. Kirilyuk, A. Kimel, O. Eriksson, and M. Pereiro, Phys. Rev. B 92, 094411 (2015).

[17] R. S. Malik, E. K. Delczeg-Czirjak, R. Knut, D. Thonig, I. Vaskivskyi, D. Phuyal, R. Gupta, S. Jana, R. Stefanuik, Y. O. Kvashnin, S. Husain, A. Kumar, P. Svedlindh, J. Soderstrom, O. Eriksson, and O. Karis, Phys. Rev. B 104, L100408 (2021).

[18] M. Battiato, K. Carva, and P. M. Oppeneer, Phys. Rev. Lett. 105, 027203 (2010).

[19] O. Eriksson, A. Bergman, L. Bergqvist, and J. Hellsvik, Atomistic Spin Dynamics: Foundations and Applications (Oxford University Press, New York, 2017).

[20] R. F. L. Evans, U. Atxitia, and R. W. Chantrell, Phys. Rev. B 91, 144425 (2015).

[21] E. Runge and E. K. U. Gross, Phys. Rev. Lett. 52, 997 (1984).

[22] The Elk Code, http://elk.sourceforge.net/.

[23] F. Willems, C. von Korff Schmising, C. Strüber, D. Schick, D. W. Engel, J. K. Dewhurst, P. Elliott, S. Sharma, and S. Eisebitt, Nat. Commun. 11, 871 (2020).

[24] D. Steil, J. Walowski, F. Gerhard, T. Kiessling, D. Ebke, A. Thomas, T. Kubota, M. Oogane, Y. Ando, J. Otto, A. Mann, M. Hofherr, P. Elliott, J. K. Dewhurst, G. Reiss, L. Molenkamp, M. Aeschlimann, M. Cinchetti, M. Munzenberg, S. Sharma, and S. Mathias, Phys. Rev. Research 2, 023199 (2020).

[25] J. K. Dewhurst, F. Willems, P. Elliott, Q. Z. Li, C. v. Korff Schmising, C. Struber, D. W. Engel, S. Eisebitt, and S. Sharma, Phys. Rev. Lett. 124, 077203 (2020).

[26] P. Elliott, T. Müller, J. K. Dewhurst, S. Sharma, and E. K. U. Gross, Sci. Rep. 6, 38911 (2016).

[27] K. Yao, F. Willems, C. von Korff Schmising, I. Radu, C. Struber, D. Schick, D. Engel, A. Tsukamoto, J. K. Dewhurst, S. Sharma, and S. Eisebitt, Phys. Rev. B 102, 100405(R) (2020).
[28] F. E. Hoare, B. Yates, and E. C. Stoner, Proc. R. Soc. London A 240, 42 (1957).

[29] P. A. Wolff, D. D. Davis, V. B. Compton, J. H. Wernick, and R. M. Bozorth, Phys. Rev. 122, 1157 (1961).

[30] G. G. Low and T. M. Holden, Proc. Phys. Soc. 89, 119 (1966).

[31] P. Mohn and K. Schwarz, J. Phys.: Condens. Matter 5, 5099 (1993).

[32] S. Iihama, Y. Sasaki, H. Naganuma, M. Oogane, S. Mizukami, and Y. Ando, J. Phys. D: Appl. Phys. 49, 035002 (2016).

[33] See Supplemental Material at http://link.aps.org/supplemental/ 10.1103/PhysRevB.105.L100401 for the convergence of the moment with respect to the number of $k$-points and time step.

[34] J. Dewhurst, K. Krieger, S. Sharma, and E. Gross, Comput. Phys. Commun. 209, 92 (2016).

[35] S. Pittalis, G. Vignale, and F. G. Eich, Phys. Rev. B 96, 035141 (2017).

[36] F. G. Eich, S. Pittalis, and G. Vignale, Phys. Rev. B 88, 245102 (2013).

[37] S. R. Acharya, V. Turkowski, G. P. Zhang, and T. S. Rahman, Phys. Rev. Lett. 125, 017202 (2020).

[38] W. S. Fann, R. Storz, H. W. K. Tom, and J. Bokor, Phys. Rev. B 46, 13592 (1992).

[39] C.-K. Sun, F. Vallée, L. H. Acioli, E. P. Ippen, and J. G. Fujimoto, Phys. Rev. B 50, 15337 (1994).

[40] C. Suárez, W. E. Bron, and T. Juhasz, Phys. Rev. Lett. 75, 4536 (1995).

[41] C. Herring, Magnetism: Exchange Interactions Among Itinerant Electrons (Academic, New York, 1966).

[42] L. Nordström and A. Mavromaras, Europhys. Lett. 49, 775 (2000).

[43] L. Sandratskii and P. Guletskii, J. Phys. F: Met. Phys. 16, L43 (1986).

[44] K. Krieger, J. Dewhurst, P. Elliott, S. Sharma, and E. Gross, J. Chem. Theory Comput. 11, 4870 (2015).

[45] S. Jana, R. S. Malik, Y. O. Kvashnin, I. L. M. Locht, R. Knut, R. Stefanuik, I. Di Marco, A. N. Yaresko, M. Ahlberg, J. Åkerman, R. Chimata, M. Battiato, J. Soderstrom, O. Eriksson, and O. Karis, Phys. Rev. Research 2, 013180 (2020). 